\title{
Carbon-Doped Boron Nitride Nanomesh: Stability and Electronic Properties of Adsorbed Hydrogen and Oxygen
}

\author{
G. C. Loh, ${ }^{*}{ }^{\dagger}, \dagger$ Sandeep Nigam, ${ }^{\S}$ G. Mallick, ${ }^{\perp}$ and Ravindra Pandey ${ }^{*}{ }^{\dagger}$ \\ ${ }^{\dagger}$ Department of Physics, Michigan Technological University, Houghton, Michigan 49931, United States \\ ${ }^{\ddagger}$ Institute of High Performance Computing, 1 Fusionopolis Way, \#16-16 Connexis, Singapore, Singapore 138632 \\ ${ }^{\S}$ Chemistry Division, Bhabha Atomic Research Centre, Trombay, Mumbai 400085, India \\ ${ }^{\perp}$ Weapons and Materials Research Directorate, U.S. Army Research Laboratory, Aberdeen Proving Ground, Maryland 21005, United \\ States
}

\section{Supporting Information}

ABSTRACT: Atomic or molecular preferential adsorption on a surface template provides a facile and feasible means of fabricating ordered low-dimensional nanostructures with tailored functionality for novel applications. In this study, we demonstrate that functionality of C-doped BN nanomesh can be tailored by an external electric field which modifies the strength of the adsorbate binding to the nanomesh. Specifically, selective binding of $\mathrm{H}, \mathrm{O}, \mathrm{H}_{2}$, and $\mathrm{O}_{2}$ at various sites of the C-doped nanomesh-within the pore, on the wire, and at an intermediate site-is investigated with density functional theory. The calculated results find that atomic species are bound, but the molecular species are not bound to the nanomesh. We have shown that it is possible to modify the adsorbate binding energy with the application of an external field, such that the molecular $\mathrm{H}_{2}$ can be bound at the pore region of the nanomesh. Interestingly, the work function of the nanomesh has a close correlation with the adsorbate binding energy with the $\mathrm{BN}$ nanomesh.

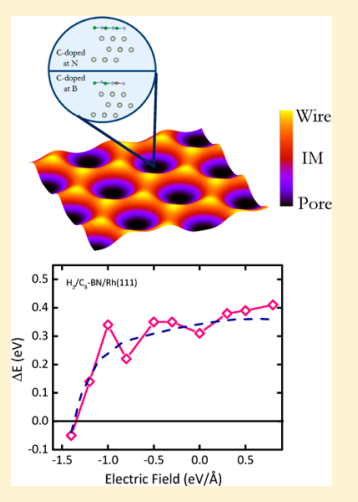

\section{INTRODUCTION}

Elevated levels of pollution from greenhouse gas emissions and urban air contaminants contribute significantly to the climate change. To a certain extent, this sparks the out-of-the-box approach in the energy industry, and draws some of its focus away from the conventional fuel sources. One of the potential alternatives is fuel cells. As these hydrogen-powered technologies have progressed in leaps and bounds in recent years, there is a growing necessity to explore novel materials or techniques for its storage in large amounts at ambient temperature and low pressure, with low volume and weight, while performing with fast kinetics involving charge-recharge processes. ${ }^{1}$ Of the numerous approaches, gas-on-solid adsorption has been identified to be particularly safe and energy-efficient. ${ }^{2}$ The solid material candidates include zeolites, ${ }^{3}$ carbon-based materials (such as activated carbon, ${ }^{2}$ carbon nanotubes, ${ }^{4}$ carbon fibers, ${ }^{5,6}$ pillared-graphene nanostructures $^{7-9}$ ), metal-organic frameworks (MOFs), ${ }^{10}$ and nanostructured metal particle or films. ${ }^{11-13}$ Notably outstanding are carbon fibers of the "platelet" and "herringbone" forms with storage capacities exceeding 50 and $60 \mathrm{wt} \%$, respectively, while other carbon structures attain at least 7 wt \% in performance. $^{2}$ The excellent storage performance of these materials makes them viable solutions for hydrogen storage. On the other hand, oxygen storage is also important in the energy industry, such as the catalytic combustion process. ${ }^{14,15}$ More significantly, the presence of oxygen-storage materials enhances the performance of proton-exchange membrane fuel cells. ${ }^{16}$
Therefore, efficiency of hydrogen and oxygen storage materials is paramount to the fuel cell technologies.

A nanomesh could be such a feasible configuration that provides the functionality of both hydrogen and oxygen storage. $^{17}$ In general, the nanomesh is a two-dimensional superstructure which comprises regions of depression called "pores" (lower region) and a continuous mesh-like structure called "wire" (higher region) in a corrugated form. ${ }^{18,19}$ Due to lattice mismatch between the nanomesh and the substrate, some of the atoms at the interface experience larger repulsive forces than others (and vice versa for attractive forces), and consequently buckling occurs on the surface. ${ }^{19}$ In such a buckled configuration, what essentially draws attention to the nanomesh from the scientific community is its ability to trap various types of molecules. Hence, a nanomesh can be seen as a candidate material for hydrogen and oxygen storage applications. This is what we will explore in this work considering a nanomesh comprised of $\mathrm{BN}$, and employ density functional theory (DFT) to investigate the selective binding (storage) of $\mathrm{H}, \mathrm{O}, \mathrm{H}_{2}$, and $\mathrm{O}_{2}$ at various sites of the nanomesh-within the pore, on the wire, and at an intermediate site. Since the doping of a $\mathrm{BN}$ sheet and its nanostructures with $\mathrm{C}$ is a typical method to control the electronic properties of these systems, ${ }^{20-22}$ we will also consider C-doped BN nanomesh. The energetics of a

Received: August 14, 2014

Revised: September 24, 2014

Published: September 25, 2014 
bare $\mathrm{BN}$ nanomesh, including the binding strength of the adsorbates to the different regions of the nanomesh, will be compared with that of a C-doped nanomesh. From its local electrostatic potential, the work function of the nanomesh will be derived, and its relationship with the binding strength of the adsorbates will be elucidated. We will also examine the effect of external electric field on the adsorbate binding energy since the adsorbed molecules are inherently a result of the polarization effect induced by surface dipoles. Last but not least, since a hydrogen binding energy within the range of -0.1 to $-0.5 \mathrm{eV} /$ atom is optimal for storage applications, ${ }^{23}$ we will then establish the feasibility of using a $\mathrm{BN}$ nanomesh for hydrogen storage.

\section{COMPUTATIONAL MODEL}

The first reported study of nanomesh is that of hexagonal boron nitride; ${ }^{18}$ however, graphene ${ }^{24-30}$ and silicon carbide ${ }^{31}$ nanomeshes are also recently investigated. The BN nanomesh was suggested to be formed by two offset layers of $\mathrm{BN}$ with 2 $\mathrm{nm}$ pores at $3 \mathrm{~nm}$ periodicity by thermally decomposing borazine on a rhodium (111) substrate. ${ }^{18}$ However, recent lowtemperature STM images revealed it to consist of a single BN layer. ${ }^{19}$ This is also verified by DFT calculations on a single layer corrugated model ${ }^{32}$ which predicts the splitting of the $\sigma$ bands seen in the experimental STM image. ${ }^{18}$ Apart from the $\mathrm{BN} / \mathrm{Rh}(111)$ nanomesh, ${ }^{32-38}$ other types of nanomesh systems with an incommensurate $\mathrm{BN} /$ metal substrate have been examined, e.g. $\mathrm{Ru}(001),{ }^{34,35,39-43} \mathrm{Pd}(110),{ }^{35,44} \mathrm{Ag}(111),{ }^{35,45}$ $\operatorname{Pt}(111),{ }^{34,35,39,46}$ and $\operatorname{Ir}(111){ }^{47,48}$ On the other hand, commensurate $\mathrm{BN} /$ metal substrate systems such as $\mathrm{BN}$ with $\mathrm{Cu}(111)^{35,49,50}$ or $\mathrm{Ni}(111)^{35,50-52}$ are not structurally corrugated, but rather reveal Moiré-like electronic corrugation. Having established the nanomesh configuration, the next imperative question concerns the nature of its ability to trap atoms or molecules. This question has been addressed previously in several investigations reporting adsorption of $\mathrm{C}_{60}{ }^{18}$ small $\mathrm{Au}$ clusters, ${ }^{53,54} \mathrm{Xe}$ atoms, ${ }^{55}$ transition-metal adatoms ( $\mathrm{Ru}, \mathrm{Rh}, \mathrm{Pt}, \mathrm{Pd}, \mathrm{Au}, \mathrm{Ni}, \mathrm{Co}, \mathrm{Fe}, \mathrm{Cu}, \mathrm{Ag}),{ }^{56,57}$ and ice clusters ${ }^{58}$ on the $\mathrm{BN}$ nanomesh. Relevant to the discussion, the electronic structure and template function of graphene and $\mathrm{BN}$ nanomesh on $\mathrm{Ru}(001)$ are compared in ref 40 .

In this work, we consider the $\mathrm{Rh}(111)$ substrate for the $\mathrm{BN}$ nanomesh. The $h-\mathrm{BN} / \mathrm{Rh}(111)$ nanomesh typically consists of a lattice formed by a $13 \times 13 \mathrm{~h}$-BN supercell deposited on a 12 $\times 12 \mathrm{Rh}(111)$ supercell (also known as a 13-on-12 supercell)..$^{32,53,54}$ In a given model, the substrate is generally simulated by the slab model containing multilayers. For example, simulation of the $\mathrm{Rh}(111)$ slab may require 3 layers containing at least 1100 atoms. Although simulation of the substrate of this scale can reasonably be handled by a large computing cluster, we realize that it is not necessary to recreate the nanomesh in its entirety. Specifically, we will consider three distinct regions of the nanomesh, namely the "pore", "intermediate region/IM", and "wire", which mimic the binding sites for the adsorption. These different regions can be modeled by using a much smaller supercell requiring relatively modest computational resources. This is similar to the approach employed to study adsorption of gold atoms on a nanomesh $^{53,54}$ where transition between pores and wires is sharp and takes place within a couple of $\mathrm{BN}$ bond lengths. It has been established that the qualitative trends and conclusions are not affected by the approximations made in modeling a small fragment of the nanomesh. ${ }^{53}$
Note that in our study, a perfect crystalline layer of $\mathrm{BN}$ is considered. In experiments, however, line defects will be formed when $\mathrm{BN}$ islands coalesce into a nanomesh. ${ }^{59}$ The magnitude of the adsorbate binding energy is likely to depend on the nature of the defect, type of species, and hence the electrostatic interaction between the defect and species. This is exemplified by two previous studies on the effect of the defect on adsorption; in the first case, ${ }^{60}$ the binding energy increases with the coordination of the defect site (i.e., the "extent" of defect), while this trend is not observed in the second study. ${ }^{61}$

In the $\mathrm{BN} / \mathrm{Rh}(111)$ configuration, $\mathrm{B}$ or $\mathrm{N}$ atoms at the center of the pore region appear to occupy the (fcc,top) configuration on rhodium atoms, ${ }^{19}$ while IM and wire regions away from the pore have a continuum of offset from the (fcc,top) sites. One can therefore consider the (fcc,top) configuration for all the regions in calculations without the loss of significant accuracy. To mimic the corrugation of the structure, a larger separation between the substrate and the sheet for the IM and wire regions relative to that optimized for the pore region was considered. The distance between the highest and lowest points ${ }^{34}$ of the nanomesh is calculated to be $1.9 \AA$. Thus, the distances of 3.13 and $4.08 \AA$ between the substrate and the sheet were taken for the IM and wire regions, respectively. The calculated binding energy of the sheet at the pore, IM, and wire regions is -1.06 , 0.33 , and $0.47 \mathrm{eV}$, respectively, suggesting the sheet to be bound at the pore region.

The supercell considered consists of commensurate $2 \times 2$ cells of $h$-BN and $\mathrm{Rh}(111)$. Three $\mathrm{ABC}$-stacked layers of rhodium atoms with the FCC structure are taken to simulate the substrate. The dopant, $\mathrm{C}$ substitutes either $\mathrm{B}$ or $\mathrm{N}$ in the $h$ $\mathrm{BN}$ sheet. Since $h$-BN and $\mathrm{Rh}(111)$ have a lattice mismatch of about $8 \%$, the $\mathrm{C}$-doped $\mathrm{BN}$ sheet is slightly stretched to match the lattice constant of $\mathrm{Rh}(111)$ in order to attain the commensurability of the $2 \times 2$ supercell.

Calculations based on DFT are performed with the VASP (Vienna $\mathrm{Ab}$ Initio Simulation Package) package, with the implementation of projector augmented-wave (PAW) pseudopotentials $^{62}$ (with an energy cutoff of $400 \mathrm{eV}$ ) and the PerdewBurke-Ernzerhof (PBE) exchange-correlation functional in the generalized gradient approximation (GGA) ${ }^{63}$ Note that we have also considered the dispersion-corrected functional form which yielded a very small change in total energy in agreement with the calculations; the van der Waals corrected DFT results are comparable to the GGA results on physisorption of molecular hydrogen on graphene and carbon nanotubes. ${ }^{64}$

The Brillouin zone is sampled with a $(10 \times 10 \times 1) \mathrm{k}$-point mesh using the Monkhorst-Pack scheme. ${ }^{65}$ The structures are optimized until the forces (as calculated by the HellmannFeynman formalism ${ }^{66,67}$ ) are less than $10^{-5} \mathrm{eV} / \AA$. In the supercell, the $\mathrm{BN} / \mathrm{Rh}(111)$ nanomesh is separated from its periodic image in the direction perpendicular to the surface by a vacuum region of $8 \AA$. Note that calculations performed with a larger vacuum region of $12 \AA$ retain the shape of the local electrostatic potential for the $\mathrm{C}$-doped $\mathrm{BN}$ nanomesh [see the Supporting Information, Figure S1]. Dipole corrections are applied in the direction perpendicular to the nanomesh surface to avoid interactions between periodically repeated images.

To partition the continuous charge density among the atoms in the system, Bader's atom in molecules theory ${ }^{68}$ is implemented, such that the atomic basin is determined at the zero flux surface around the atom. This surface is observed in the two-dimensional sense at which the charge density is at a minimum perpendicular to the system surface. Therefore, the 


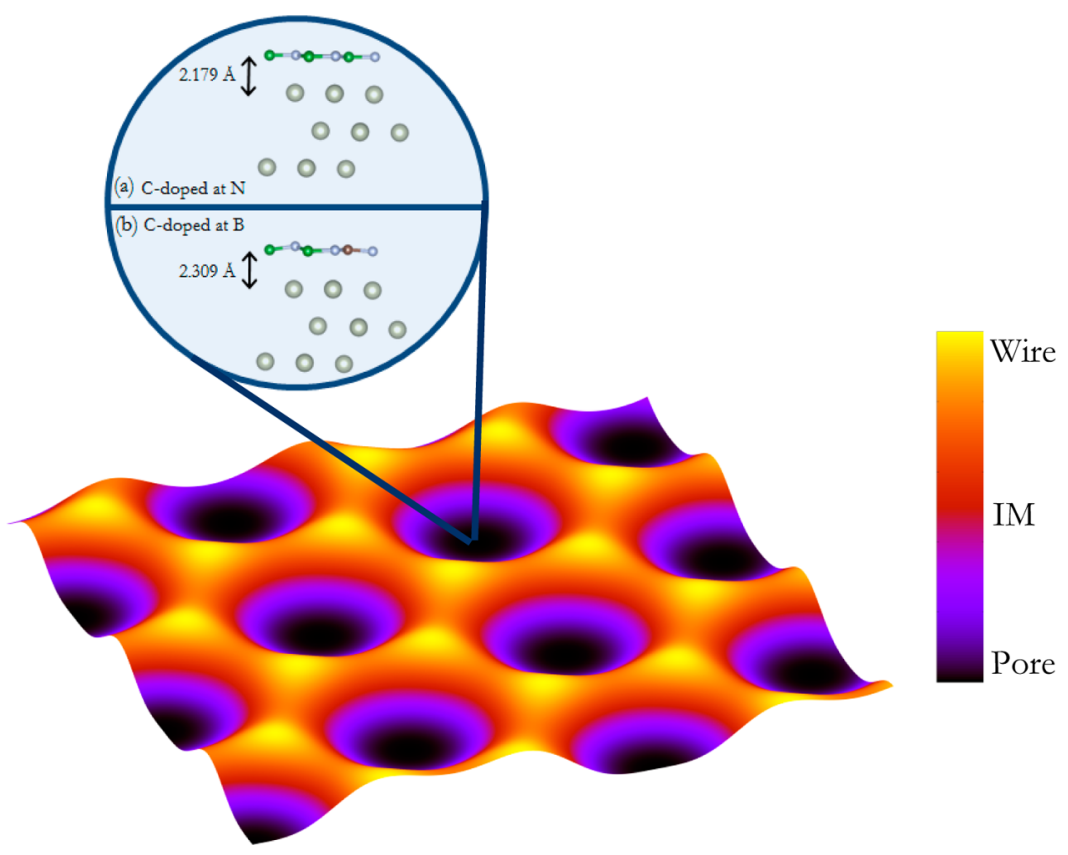

Figure 1. $\mathrm{BN} / \mathrm{Rh}(111)$ nanomesh in the $(\mathrm{B}, \mathrm{N})=\left(\mathrm{fcc}_{\mathrm{Rh}}, \mathrm{top}_{\mathrm{Rh}}\right)$ configuration. The side view is shown in the inset: (a) C is doped at the $\mathrm{N}$ site; $(\mathrm{b})$ $\mathrm{C}$ is doped at the $\mathrm{B}$ site. Atoms in gray are $\mathrm{B}$, green are $\mathrm{N}$, and purple are $\mathrm{C}$.

total electronic charge of each atom can be defined, and the charge among the atoms distributed.

\section{RESULTS AND DISCUSSION}

The atomic structure of the nanomesh is generally determined by interplay between the interaction of $\mathrm{Rh}$ with $\mathrm{B}$ or $\mathrm{N}$ atoms and the interaction within the sheet. Figure 1 shows the optimized configuration of the BN nanomesh. Similar to what was reported earlier, ${ }^{32,34,51}$ the sheet is buckled in such a way that $\mathrm{N}$ atoms are pushed further away from the Rh substrate, whereas B atoms are pulled toward it. Substitution of $\mathrm{C}$ in the sheet does not change its separation with the substrate. However, degree of buckling is disparate in the $C_{N}$ and $C_{B}$ configurations of the nanomesh-the mean height of $\mathrm{N}$ atoms is higher than that of $\mathrm{B}$ atoms by approximately $0.05 \AA$ for $\mathrm{C}_{\mathrm{N}}$, while it is $0.14 \AA$ for $\mathrm{C}_{\mathrm{B}}$. Both atomic adsorbates $\mathrm{H}$ and $\mathrm{O}$ are positioned at the top of the dopant with the bond distances of $1.149,1.148,1.509$, and $1.464 \AA$ for $\mathrm{H}-\mathrm{C}_{\mathrm{N}}, \mathrm{H}-\mathrm{C}_{\mathrm{B}}, \mathrm{O}-\mathrm{C}_{\mathrm{N}}$, and $\mathrm{O}-\mathrm{C}_{\mathrm{B}}$ bonds, respectively (see the Supporting Information, Figure S2). It is to be noted here that the $\mathrm{C}-\mathrm{H}$ and $\mathrm{C}-\mathrm{O}$ bond lengths are reported ${ }^{69}$ to be 1.06 and $1.43 \AA$, respectively.

3.1. Energetics. The calculated binding energy of the atomic and molecular adsorbates at the pore, IM, and wire regions of the nanomesh are listed in Tables S1 and S2 [see the Supporting Information]. The results are also summarized in Figure 2. The binding energy of adsorbate can be expressed as

$$
\Delta E=E_{\mathrm{ads} / \mathrm{C}-\mathrm{BN} / \mathrm{Rh}}-E_{\mathrm{C}-\mathrm{BN} / \mathrm{Rh}}-E_{\mathrm{ads}}
$$

where $E_{\mathrm{ads} / \mathrm{C}-\mathrm{BN} / \mathrm{Rh}}, E_{\mathrm{C}-\mathrm{BN} / \mathrm{Rh}}$, and $E_{\mathrm{ads}}$ are the total energies of the structure with adsorbate, without adsorbate, and the adsorbate, respectively. Thus, a bound atom/molecule will be associated with a negative binding energy (eq 1). Note that the energies of the adsorbates were calculated by the spin-polarized electronic structure calculations.

We find $\mathrm{H}$ to be bound for all the regions of $\mathrm{C}$-doped nanomesh. This is not the case for the undoped BN nanomesh where atomic $\mathrm{H}$ can only be bound at the $\mathrm{N}$ site for the pore

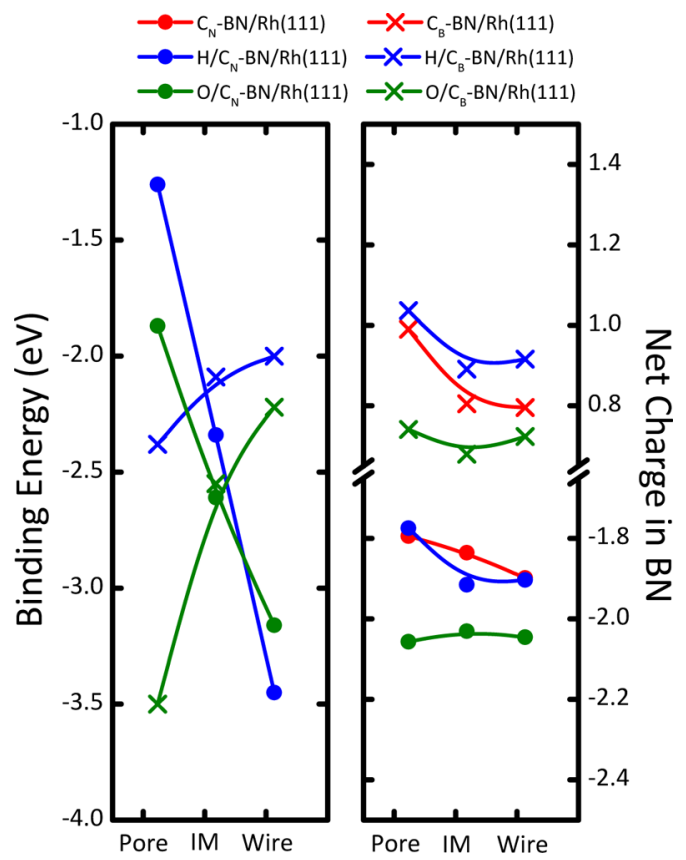

Figure 2. C-doped BN nanomesh: binding energy of the atomic and molecular adsorbates and Bader's charge associated with the BN sheet for pore, IM, and wire regions of the nanomesh.

and IM regions (Table S1, Supporting Information). The calculated results therefore suggest that the bond between $\mathrm{H}$ and the dopant $\mathrm{C}$ is relatively stronger than that of $\mathrm{H}$ and $\mathrm{B} / \mathrm{N}$ of the undoped nanomesh. Interestingly, the preferred adsorbate binding site is predicted to depend on the region of the nanomesh; $\mathrm{H}$ adsorption prefers the $\mathrm{C}_{\mathrm{N}}$ site at the wire region, while the $C_{B}$ site is preferred at the pore region. Note that a molecular $\mathrm{H}_{2}$ configuration either perpendicular or parallel to surface is predicted by our calculations to be unbound to the nanomesh (Table S1, Supporting Information). This is in contrast to the case of the pristine $\mathrm{BN}$ nanotube 
whereby the binding energy of $\mathrm{H}_{2}$ is predicted to be $-0.09 \mathrm{eV} /$ atom. ${ }^{70}$ Akin to that of $\mathrm{H}$, the $\mathrm{C}-\mathrm{O}$ bond is generally stronger than the $\mathrm{B}-\mathrm{O}$ and $\mathrm{N}-\mathrm{O}$ bonds and $\mathrm{C}_{\mathrm{B}}$-doped nanomesh binds $\mathrm{O}$ more strongly at the pore region (Table S2, Supporting Information). The $\mathrm{O}_{2}$ molecule with a bond length of $1.4 \AA$ could not be trapped by the $\mathrm{C}$-doped nanomesh at any site including the perpendicular and/or parallel orientations (Table S2, Supporting Information).

3.2. Work Function. The binding energy of atomic and molecular species adsorbed on the nanomesh is expected to be determined by the work function of the C-doped $\mathrm{BN}$ nanomesh. Since adsorption occurs on the surface of the nanomesh, the binding energy of the adsorbed species is primarily governed by the nature of the nanomesh; the choice of substrate, and hence the lattice mismatch between the substrate and grown layer, may have only a small effect on the adsorbate binding energy. Note that the work function is defined as the minimum energy required to remove an electron from the bulk of the material to the vacuum level, and is essentially termed as the electron binding energy. By averaging the electrostatic potential across the horizontal plane (parallel to the surface of the nanomesh), and plotting it along the

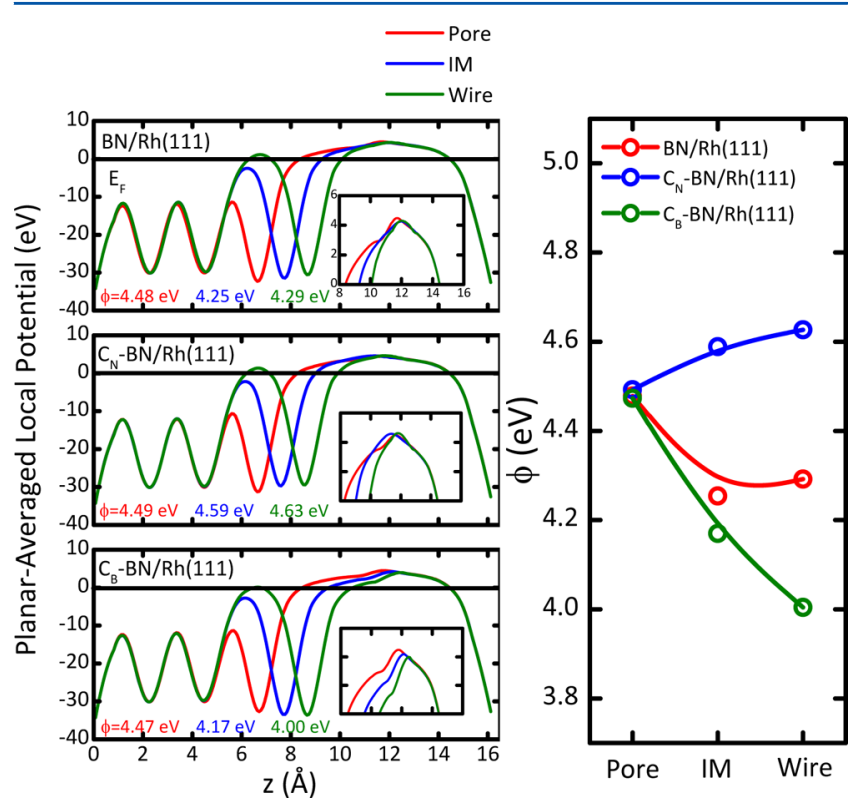

Figure 3. Calculated planar-averaged local potential and the work function of the BN nanomesh. The insets in the left pane show a magnified view of the local potential curve near the peak.

direction normal to the surface (Figure 3), the work function of the nanomesh can be calculated as

$$
\phi=E_{\mathrm{vac}}-E_{\mathrm{F}}
$$

where $E_{\mathrm{vac}}$ is the vacuum level, and $E_{\mathrm{F}}$ is the Fermi energy. Since the planar-averaged local potential is adjusted to the Fermi energy such that $E_{\mathrm{F}}=0$, the work function can then be obtained from the vacuum level as shown in Figure 3.

The work function of an undoped nanomesh is approximately $4.30 \mathrm{eV}$, which compares well with that $(4.15 \mathrm{eV})$ reported in ref 71 . At the pore region of the nanomesh, regardless of the presence of adsorbate, the work function is invariant. At other regions, it diverges; a higher work function for the wire region of $\mathrm{C}_{\mathrm{N}}$-doped nanomesh makes the removal of electrons into the vacuum relatively more difficult than the corresponding removal from the pore region (Figure 3 ). This is consonant with the trend of change of binding energy of atomic $\mathrm{H}$ and $\mathrm{O}$ to the nanomesh (Tables S1(b) and S2(b), Supporting Information); there is a direct relationship between the binding energy and work function of the nanomesh. Likewise, this correlation is also seen in $\mathrm{C}_{\mathrm{B}}$-doped nanomesh. The sitedependent nature of the work function reflects the description given in ref 33 , whereby the work function at the wire region is greater than that at the pore region.

3.3. Chemical Bonding. To understand the regiondependent variation in the binding energy of the adsorbates considered (Figure 2), the role of the Rh substrate will now be examined via its interaction with the sheet at the pore, IM, and wire regions of the nanomesh. First we will look into the possibility of charge transfer between the doped sheet and the substrate, and then calculate the density of states to investigate the extent of hybridization of molecular orbitals of the $\mathrm{BN}$ sheet and the Rh substrate in the nanomesh configuration.

Considering that doping-induced effective charge in the $\mathrm{BN}$ sheet may influence the site-preference for atomic and molecular species considered, we define the net charge dQ associated with the $\mathrm{BN}$ sheet as the difference between $\mathrm{Q}$ (Cdoped sheet) and $\mathrm{Q}^{\prime}$ (C-doped nanomesh, i.e. C-doped sheet interacting with the substrate) with $Q$ being Bader's charge. ${ }^{68}$ $\mathrm{dQ}$ is expected to vary with the distance between the sheet and the $\mathrm{Rh}$ substrate in the corrugated nanomesh representing the pore, IM, and wire regions, and the negative value of dQ implies that the electronic charge is transferred from the sheet to the substrate. For the case of the $\mathrm{C}_{\mathrm{N}}$-doped sheet, $\mathrm{dQ}$ increases with the separation, i.e. more electrons are depleted. For the case of the $\mathrm{C}_{\mathrm{B}}$-doped sheet, an increase in the separation of the sheet and substrate decreases the transfer of charge to $\mathrm{BN}$, since fewer electrons are transferred from the substrate to the sheet. The charge transfer in the oxygenadsorbed nanomesh is impervious to the separation for both $\mathrm{C}_{\mathrm{N}}$-doped and $\mathrm{C}_{\mathrm{B}}$-doped sheets. This is not the case with atomic $\mathrm{H}$ where variation in the charge transfer with the pore, IM, and wire regions is predicted (Figure 2).

Bonding between the $\mathrm{C}$-doped $\mathrm{BN}$ sheet and the $\mathrm{Rh}$ substrate appears to be dominated by the hybridization of $\mathrm{Rh}$ $\mathrm{d}_{\mathrm{z}}{ }^{2}$ with $\mathrm{N}-\mathrm{p}_{z}$ orbitals. Figure 4 shows a representative case of the pore region where such hybridization induces a band near Fermi energy in the projected density of states. This band shows a small shift to the higher energies in going from the

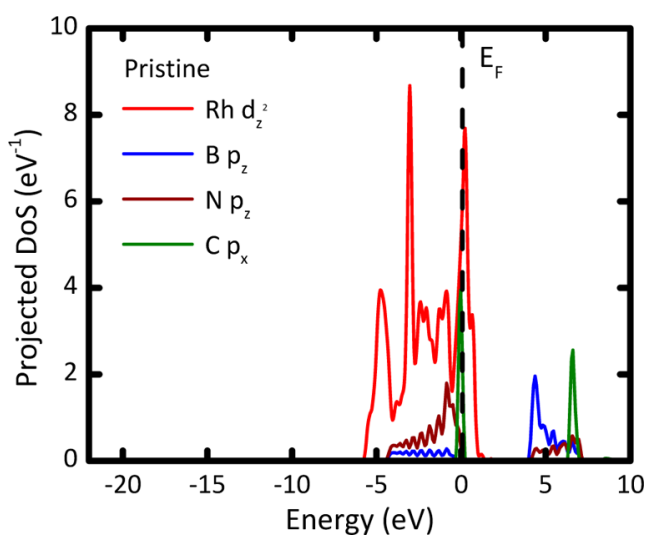

Figure 4. Projected density of states of C-doped nanomesh at the pore region. Zero is taken to be the Fermi energy of the nanomesh. 


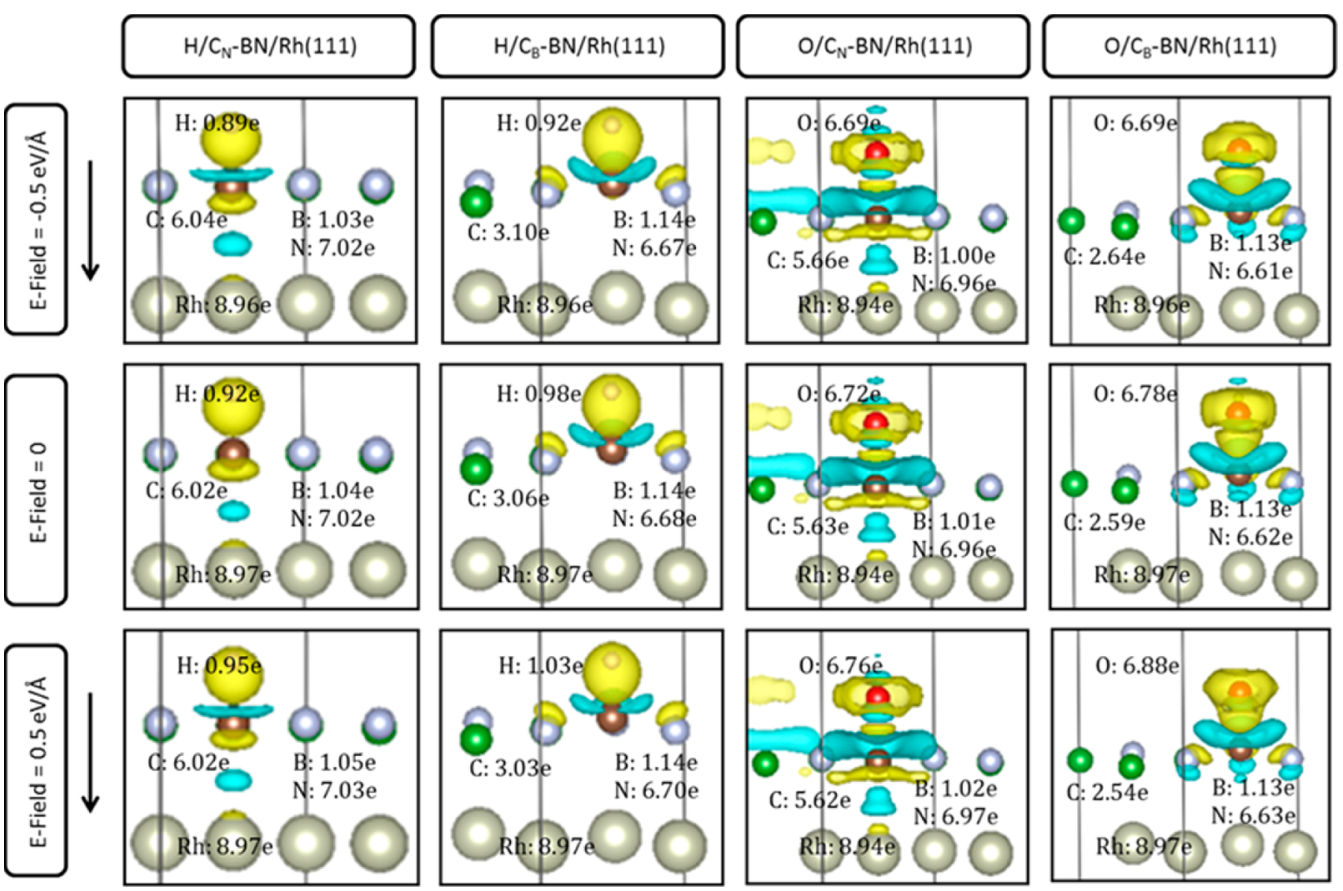

Figure 5. Pore region of the BN nanomesh: charge density difference plots projected at the interface with the isovalue of 0.00936 e/ $\AA^{3}$. Yellow: accumulation region; blue: depletion region. Atoms in gray are $\mathrm{Rh}$, green are $\mathrm{B}$, and white are $\mathrm{N}$. "eV/ $\mathrm{A}$ " is the unit for the electric field strength in the VASP package.

pore to IM to wire regions (see the Supporting Information, Figure S6), though the degree of shift depends on the doping site-it is larger for $\mathrm{C}_{\mathrm{N}}$-doped relative to $\mathrm{C}_{\mathrm{B}}$-doped nanomesh. This disparity is due to the dissimilitude in the extent of charge transfer in the nanomesh; $\mathrm{dQ}$ is larger for $\mathrm{C}_{\mathrm{N}}$-doped than for $\mathrm{C}_{\mathrm{B}}$-doped for all regions of the nanomesh (Figure 2). Note that variation of energy states at different regions in the adsorbate/ C-doped nanomesh configuration is akin to that without adsorption (see the Supporting Information, Figures S5 and S6), and is again well-comprehended by the variation in $d Q$ shown in Figure 2.

3.4. Tuning of Adsorbate Binding Energy via External Electric Field. The confining potential in the pore region is largely ascribed to the presence of surface dipoles which are formed due to the polarization effect by the metal substrate. ${ }^{33}$ These dipoles can trap molecules or small clusters that are highly polarizable. Therefore, the binding strength of the adsorbate is closely related to the surface dipole moment of the $\mathrm{BN}$ nanomesh. Considering that the dipole moment is a measure of the separation of positive and negative charges, application of an external electric field in the direction perpendicular to the nanomesh surface can modulate the surface dipole moment, thereby tailoring the adsorption. This essentially permits the tuning of the "capture and release" processes in the energy storage applications. In relevance to the above discussion, the band gaps of $\mathrm{BN}$ sheets are relatively sensitive to the magnitude of the external electric field. ${ }^{72,73}$

The charge transfer at the pore region of the nanomesh with the adsorbates is examined in Figure 5 where the charge density difference plot shows accumulated and depleted regions at the interface of the nanomesh. The electric field is applied in the direction perpendicular to the nanomesh surface. For atomic $\mathrm{H}$ adsorbed on $\mathrm{C}_{\mathrm{N}}$-doped nanomesh, $\mathrm{H}, \mathrm{C}$, and $\mathrm{Rh}$ atoms determine the bonding characteristics at the interface. There is minimal charge transfer between $\mathrm{H}$ and $\mathrm{B}$ atoms (Figure 5).
This is in stark contrast to the case of $\mathrm{C}_{\mathrm{B}}$-doped nanomesh where, in addition to the $\mathrm{C}-\mathrm{H}$ charge transfer, accumulation and depletion regions between $\mathrm{H}$ and neighboring $\mathrm{N}$ atoms (in white) are considerably large, indicating a non-negligible charge transfer between $\mathrm{H}$ and $\mathrm{N}$ at the interface. For the case of adsorption of atomic $\mathrm{O}$, the charge transfer occurs between $\mathrm{B}-$ $\mathrm{O}$ and $\mathrm{N}-\mathrm{O}$ atoms in $\mathrm{C}_{\mathrm{N}}$-doped and $\mathrm{C}_{\mathrm{B}}$-doped nanomesh, respectively, while the $\mathrm{Rh}-\mathrm{C}$ charge transfer occurs only in $\mathrm{C}_{\mathrm{N}^{-}}$ doped nanomesh.

By using Bader's method to draw the atomic boundaries, ${ }^{68}$ change in the mean charge of each constituent element with the electric field strength is probed. In all cases, atoms, with the exception of the dopant, accumulate charges with the increase of the positive field strength. When the electric field is increased in the reverse direction, charges are depleted. The degree of change of charge transfer is much higher in $\mathrm{C}_{\mathrm{B}}$-doped nanomesh, suggesting that electron transfer is sensitive to the electric field strength for $\mathrm{C}_{\mathrm{B}}$-doped nanomesh, though fewer charges are transferred at all sites under zero bias (Figure 2). For instance, variation of the field from -0.5 to $0.5 \mathrm{eV} / \AA$ induces changes in the atomic charges; the change is about $7 \%$ for $\mathrm{H}$ and $1 \%$ for $\mathrm{O}$ in $\mathrm{C}_{\mathrm{N}^{-}}$-doped nanomesh, and $12 \%$ for $\mathrm{H}$ and $3 \%$ for $\mathrm{O}$ in $\mathrm{C}_{\mathrm{B}}$-doped nanomesh.

Figure 6 shows variation of the adsorbate binding energy $(\Delta E)$ with the electric field for the pore, IM, and wire regions of the nanomesh. In all cases, the adsorbate binding energy of atomic $\mathrm{O}$ is larger than that of atomic $\mathrm{H}$. A direct relationship between variations in $\Delta E$ with the electric field is not seen, though both adsorbates have a unique response to the electric field at the wire region of the $\mathrm{C}_{\mathrm{N}}$-doped nanomesh, as compared to that associated with the other two regions including pore and IM regions. That notwithstanding, the trend in each scenario is consistent with the zero-field values of $\Delta E$ given in Tables $\mathrm{S} 1$ and S2, Supporting Information. The degree of change of $\Delta E$ in going from -0.5 to $0.5 \mathrm{eV} / \AA$ (Table S3, 


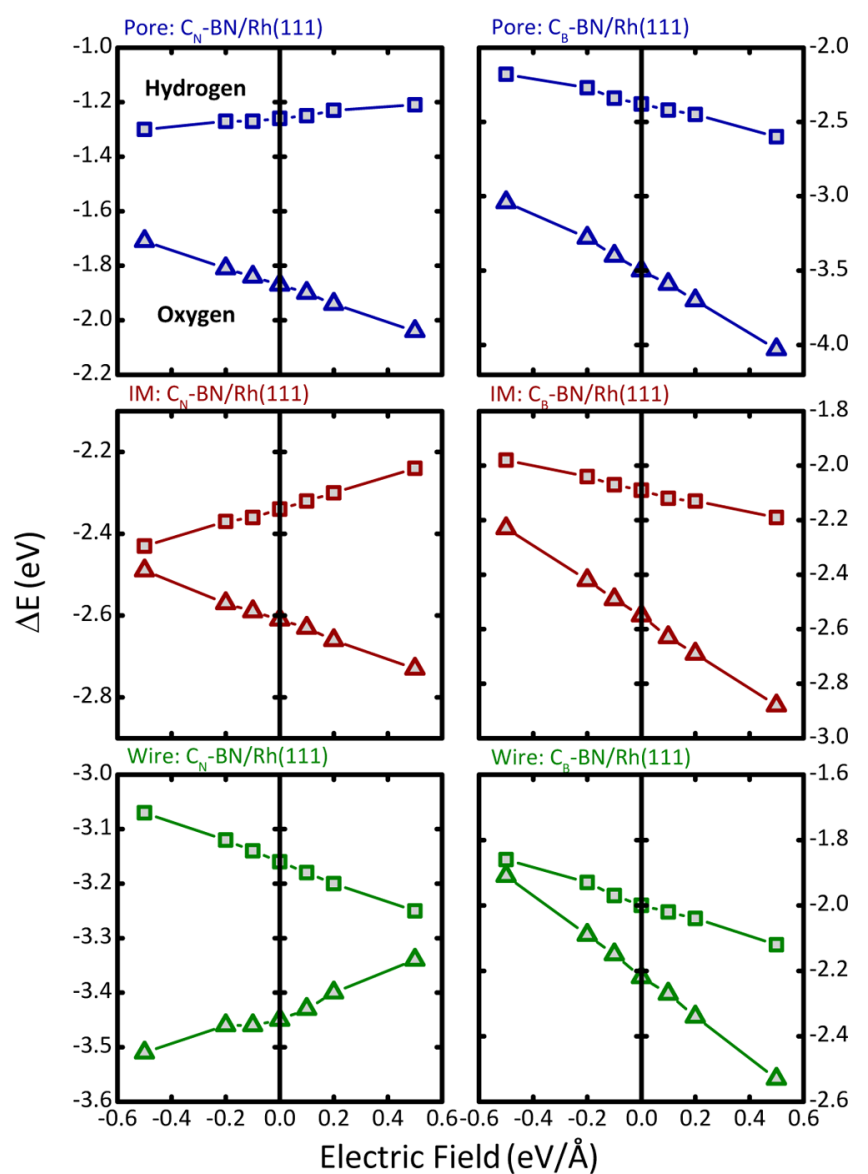

Figure 6. A variation in the adsorbate binding energy $(\Delta E)$ with the electric field at the pore, IM, and wire regions of the C-doped nanomesh.

Supporting Information) demonstrates that $C_{B}$-doped nanomesh is more responsive to variation in the strength of the electric field considered here. Note that $\mathrm{eV} / \AA$ is the unit for the electric field strength in the VASP program (see the Supporting Information, page S-13). This prediction is consistent with the prediction of a relatively large transfer of charge occurring for the $C_{B}$-doped nanomesh under the external electric field (Figure 5).

The ability to alter the binding energy of the adsorbates on $\mathrm{BN}$ nanomesh by the external electric field suggests that atoms which are not bound to the nanomesh under normal circumstances might be able to bind to the nanomesh when an electric field is applied. This is what we have seen: application of an external field finds the molecular $\mathrm{H}_{2}$ to be bound at the pore region and $\Delta E$ becomes negative for the molecular $\mathrm{H}_{2}$ in $\mathrm{C}_{\mathrm{B}}$-doped nanomesh at $-1.4 \mathrm{eV} / \AA$ (Figure 7 ). Interestingly, $\mathrm{C}_{\mathrm{N}}$-doped nanomesh shows an opposite trend in $\Delta E$ which becomes positive at $-1.4 \mathrm{eV} / \AA \AA$. Note that such a high electric field has been reported in practice, for example in scanning tunnelling microscopy (STM) applications. ${ }^{74}$

To ascertain the origin of the disparity between the binding strengths of $\mathrm{H}_{2}$ with $\mathrm{C}_{\mathrm{N}}$-doped and $\mathrm{C}_{\mathrm{B}}$-doped nanomeshes, we examine their projected band structures at the electric field strength of $-1.4 \mathrm{eV} / \AA$. . Note that the charge of the molecular adsorbate does not vary with the electric field, suggesting that the charge transfer is not likely to be the dominant factor in determining the magnitude of the adsorption binding energy (see the Supporting Information, Figure S8).

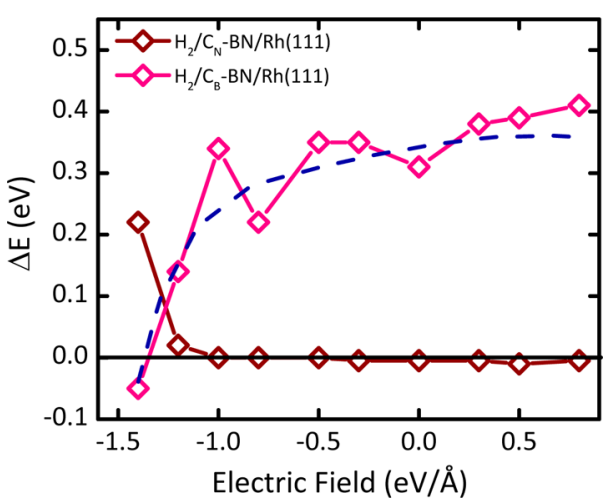

Figure 7. A variation of the adsorbate binding energy $(\Delta E)$ with the electric field for the $\mathrm{C}$-doped nanomesh at the pore region.

Figure 8 shows the projected band structure at an applied electric field of $-1.4 \mathrm{eV} / \AA$ for $\mathrm{H}_{2}$ adsorbed on C-doped $\mathrm{BN}$

(a)

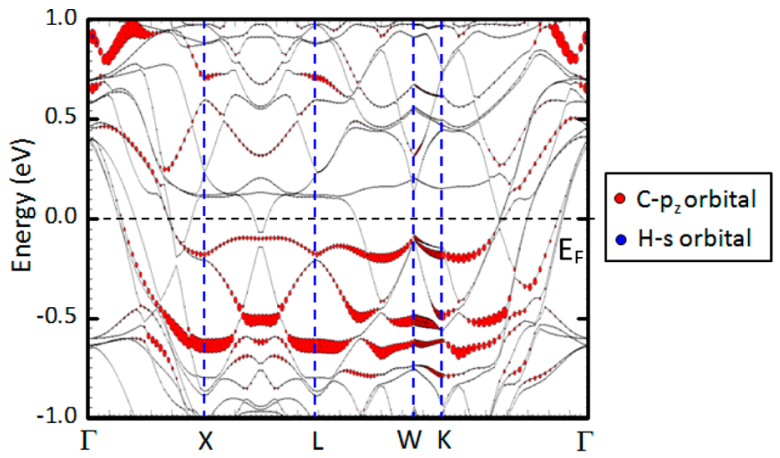

(b)

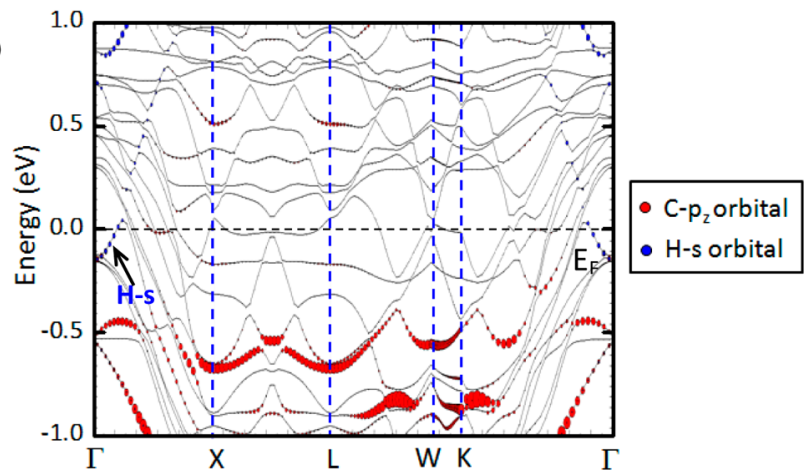

Figure 8. The projected band structure at an applied electric field of $-1.4 \mathrm{eV} / \AA ̊$ : $\mathrm{H}_{2}$ adsorbed on (a) $\mathrm{C}_{\mathrm{N}}$-doped and (b) $\mathrm{C}_{\mathrm{B}}$-doped nanomesh. The $\mathrm{C}-\mathrm{p}_{z}$ and $\mathrm{H}$-s orbitals are represented by red and blue circles, respectively.

nanomesh. Note that $\mathrm{C}-\mathrm{p}_{z}$ and $\mathrm{H}$-s orbital states are the prevailing ones that are involved in the formation of the $\mathrm{C}-\mathrm{H}$ bond. A strong hybridization of these states at $\Gamma$ in the occupied bands for $\mathrm{C}_{\mathrm{B}}$-doped nanomesh induces a large binding energy between the dopant and adsorbate. This is not the case with $\mathrm{C}_{\mathrm{N}}$-doped nanomesh. Overall, Figures 6-8 are coherent in demonstrating the feasibility of tuning the binding energy of adsorbates in the C-doped $\mathrm{BN}$ nanomeshes considered.

\section{SUMMARY}

The BN nanomesh is a corrugated form of the two-dimensional $\mathrm{BN}$ sheet that is synthesized by self-assembly on certain types 
of transition metal substrates. It has demonstrated a unique ability to trap molecules and clusters, thereby allowing it to function as a surface template in applications such as hydrogen storage at nanoscale. In this study, first-principles calculations with density functional theory are carried out to explore the ability of the nanomesh to trap atomic and molecular species of hydrogen and oxygen. The most energetically preferential site of adsorption in C-doped nanomesh is dependent on the type of atomic species ( $\mathrm{B}$ or $\mathrm{N}$ ) that is substituted by the dopant in the lattice. Atomic hydrogen and oxygen can be bound to Cdoped nanomesh, and molecular species which are arranged either perpendicular or parallel to the nanomesh surface remain unbound. For atomic adsorption, the bond between the adsorbate and the dopant is stronger than that between the adsorbate and one of the constituent atoms of the nanomesh. The work function of the BN nanomesh has a strong and direct relationship with the binding energy of adsorbates, and is contingent on the (1) type of configuration (nitrogen- or boron-substituted) and (2) site of adsorption. Furthermore, the charge transfer in $\mathrm{C}_{\mathrm{B}}$-doped nanomesh is more sensitive to the electric field strength than that seen for $\mathrm{C}_{\mathrm{N}}$-doped nanomesh. We have shown that it is possible to modify the adsorbate binding energy with the external electric field applied to $\mathrm{BN}$ nanomesh. As an ending note, we find that the BN nanomesh is not likely a candidate material for hydrogen storage applications, since the hydrogen binding energies are predicted to be beyond the optimal range of between -0.1 and $-0.5 \mathrm{eV} /$ atom.

\section{ASSOCIATED CONTENT}

\section{(S) Supporting Information}

Modeling parameters, optimized nanomesh configurations, energetics, electronic band structures, density of states, surface dipole moments, net charges, and a note on the magnitude of electric field applied. This material is available free of charge via the Internet at http://pubs.acs.org.

\section{AUTHOR INFORMATION}

\section{Corresponding Authors}

*E-mail: jgloh@mtu.edu (G.C.L.).

*E-mail: pandey@mtu.edu (R.P.).

\section{Notes}

The authors declare no competing financial interest.

\section{ACKNOWLEDGMENTS}

G.C.L. gratefully acknowledges A*STAR for funding under the A*STAR International Fellowship (2013-2015). The authors thank D. R. Banyai for his help with one of the figures. The computations were performed on the MTU Superior cluster, and support from Dr. S. Gowtham is appreciated.

\section{REFERENCES}

(1) Berry, G. D.; Aceves, S. M. Onboard storage alternatives for hydrogen vehicles. Energy Fuels 1998, 12, 49-55.

(2) Dillon, A. C.; Heben, M. J. Hydrogen storage using carbon adsorbents: past, present and future. Appl. Phys. A: Mater. Sci. Process. 2001, 72, 133-142.

(3) Yoon, J.-H. Pressure-dependent hydrogen encapsulation in Na12zeolite A. J. Phys. Chem. 1993, 97, 6066-6068.

(4) Dillon, A. C.; Jones, K. M.; Bekkedahl, T. A.; Kiang, C. H.; Bethune, D. S.; Heben, M. J. Storage of hydrogen in single-walled carbon nanotubes. Nature 1997, 386, 377-379.
(5) Tibbetts, G. G.; Meisner, G. P.; Olk, C. H. Hydrogen storage capacity of carbon nanotubes, filaments, and vapor-grown fibers. Carbon 2001, 39, 2291-2301.

(6) Park, C.; Anderson, P. E.; Chambers, A.; Tan, C. D.; Hidalgo, R.; Rodriguez, N. M. Further studies of the interaction of hydrogen with graphite nanofibers. J. Phys. Chem. B 1999, 103, 10572-10581.

(7) Varshney, V.; Patnaik, S. S.; Roy, A. K.; Froudaki, G.; Farmer, B. L. Modeling of thermal transport in pillared-graphene architectures. ACS Nano 2010, 4, 1153-1161.

(8) Loh, G. C.; Teo, E. H. T.; Tay, B. K. Interpillar phononics in pillared-graphene hybrid nanostructures. J. Appl. Phys. 2011, 110, 083502.

(9) Loh, G. C.; Teo, E. H. T.; Tay, B. K. Tuning the Kapitza resistance in pillared-graphene nanostructures. J. Appl. Phys. 2012, 111, 013515.

(10) Rosi, N. L.; Eckert, J.; Eddaoudi, M.; Vodak, D. T.; Kim, J.; O'Keeffe, M.; Yaghi, O. M. Hydrogen storage in microporous metalorganic frameworks. Science 2003, 300, 1127-1129.

(11) Hou, Y.; Kondoh, H.; Ohta, T.; Gao, S. Size-controlled synthesis of nickel nanoparticles. Appl. Surf. Sci. 2005, 241, 218-222.

(12) Hou, P. X.; Yamazaki, T.; Orikasa, H.; Kyotani, T. An easy method for the synthesis of ordered microporous carbons by the template technique. Carbon 2005, 43, 2624-2627.

(13) Bobet, J. L.; Grigorova, E.; Khrussanova, M.; Khristov, M.; Stefanov, P.; Peshev, P.; Radev, D. Hydrogen sorption properties of graphite-modified magnesium nanocomposites prepared by ballmilling. J. Alloys Compd. 2004, 366, 298-302.

(14) Song, Q.; Liu, W.; Bohn, C. D.; Harper, R. N.; Sivaniah, E.; Scott, S. A.; Dennis, J. S. A high performance oxygen storage material for chemical looping processes with $\mathrm{CO}_{2}$ capture. Energy Environ. Sci. 2013, 6, 288-298.

(15) Sugiura, M. Oxygen storage materials for automotive catalysts: ceria-zirconia solid solutions. Catal. Surv. Asia 2003, 7, 77-87.

(16) Xu, Z.; Qi, Z.; Kaufman, A. Effect of oxygen storage materials on the performance of proton-exchange membrane fuel cells. J. Power Sources 2003, 115, 40-43.

(17) A conventional nanomesh system is comprised of a thin film deposited on a metal substrate. The larger mass of such a system reduces its portability in energy applications. However, it can still be a potential material in large-scale devices where portability and mobility are significantly less crucial.

(18) Corso, M.; Auwärter, W.; Muntwiler, M.; Tamai, A.; Greber, T.; Osterwalder, J. Boron nitride nanomesh. Science 2004, 303, 217-220.

(19) Berner, S.; Corso, M.; Widmer, R.; Groening, O.; Laskowski, R.; Blaha, P.; Schwarz, K.; Goriachko, A.; Over, H.; Gsell, S.; et al. Boron nitride nanomesh: functionality from a corrugated monolayer. Angew. Chem., Int. Ed. 2007, 46, 5115-5119.

(20) Berseneva, N.; Krasheninnikov, A. V.; Nieminen, R. M. Mechanisms of postsynthesis doping of boron nitride nanostructures with carbon from first-principles simulations. Phys. Rev. Lett. 2011, $107,035501$.

(21) Beheshtian, J.; Sadeghi, A.; Neek-Amal, M.; Michel, K. H.; Peeters, F. M. Induced polarization and electronic properties of carbon-doped boron nitride nanoribbons. Phys. Rev. B 2012, 86, 194533.

(22) Berseneva, N.; Gulans, A.; Krasheninnikov, A. V.; Nieminen, R. $\mathrm{M}$. Electronic structure of boron nitride sheets doped with carbon from first-principles calculations. Phys. Rev. B 2013, 87, 035404.

(23) Walker, G., Ed. Solid-state hydrogen storage: materials and chemistry; CRC Press: Boca Raton, FL, 2008.

(24) Naumov, I. I.; Bratkovsky, A. M. Semiconducting graphene nanomeshes. Phys. Rev. B 2012, 85, 201414(R).

(25) Şahin, H.; Ciraci, S. Structural, mechanical, and electronic properties of defect-patterned graphene nanomeshes from first principles. Phys. Rev. B 2011, 84, 035452.

(26) Sako, R.; Hasegawa, N.; Tsuchiya, H.; Ogawa, M. Computational study on band structure engineering using graphene nanomeshes. J. Appl. Phys. 2013, 113, 143702. 
(27) Liu, J.; Cai, H.; Yu, X.; Zhang, K.; Li, X.; Li, J.; Pan, N.; Shi, Q.; Luo, Y.; Wang, X. Fabrication of graphene nanomesh and improved chemical enhancement for Raman spectroscopy. J. Phys. Chem. C 2012, 116, 15741-15746.

(28) Wang, M.; Fu, L.; Gan, L.; Zhang, C.; Rümmeli, M.; Bachmatiuk, A.; Huang, K.; Fang, Y.; Liu, Z. CVD growth of large area smooth-edged graphene nanomesh by nanosphere lithography. Sci. Rep. 2013, 3, 1238.

(29) Akhavan, O. Graphene nanomesh by $\mathrm{ZnO}$ nanorod photocatalysts. ACS Nano 2010, 4, 4174-4180.

(30) Standop, S.; Lehtinen, O.; Herbig, C.; Lewes-Malandrakis, G.; Craes, F.; Kotakoski, J.; Michely, T.; Krasheninnikov, A. V.; Busse, C. Ion impacts on graphene/ $\operatorname{Ir}(111)$ : interface channeling, vacancy funnels, and a nanomesh. Nano Lett. 2013, 13, 1948-1955.

(31) Chen, W.; Zhang, H. L.; Xu, H.; Tok, E. S.; Loh, K. P.; Wee, A. T. S. $\mathrm{C}_{60}$ on SiC nanomesh. J. Phys. Chem. B 2006, 110, 21873-21881. (32) Laskowski, R.; Blaha, P.; Gallauner, T.; Schwarz, K. Single-layer model of the hexagonal boron nitride nanomesh on the $\mathrm{Rh}(111)$ Surface. Phys. Rev. Lett. 2007, 98, 106802.

(33) Dil, H.; Lobo-Checa, J.; Laskowski, R.; Blaha, P.; Berner, S.; Osterwalder, J.; Greber, T. Surface trapping of atoms and molecules with dipole rings. Science 2008, 319, 1824-1826.

(34) Laskowski, R.; Blaha, P. Ab initio study of h-BN nanomeshes on $\mathrm{Ru}(001), \mathrm{Rh}(111)$, and Pt(111). Phys. Rev. B 2010, 81, 075418.

(35) Laskowski, R.; Blaha, P.; Schwarz, K. Bonding of hexagonal BN to transition metal surfaces: an ab initio density-functional theory study. Phys. Rev. B 2008, 78, 045409.

(36) Widmer, R.; Berner, S.; Gröning, O.; Brugger, T.; Osterwalder, J.; Greber, T. Electrolytic in situ STM investigation of h-BNnanomesh. Electrochem. Commun. 2007, 9, 2484-2488.

(37) Dong, G.; Fourré, E. B.; Tabak, F. C.; Frenken, J. W. M. How boron nitride forms a regular nanomesh on $\mathrm{Rh}(111)$. Phys. Rev. Lett. 2010, 104, 096102.

(38) Müller, F.; Hüfner, S.; Sachdev, H. Epitaxial growth of boron nitride on a $\mathrm{Rh}(111)$ multilayer system: formation and fine tuning of a BN-nanomesh. Surf. Sci. 2009, 603, 425-432.

(39) Preobrajenski, A. B.; Nesterov, M. A.; Ng, M. L.; Vinogradov, A. S.; Mårtensson, N. Monolayer h-BN on lattice-mismatched metal surfaces: on the formation of the nanomesh. Chem. Phys. Lett. 2007, 446, 119-123.

(40) Brugger, T.; Günther, S.; Wang, B.; Dil, J. H.; Bocquet, M.-L.; Osterwalder, J.; Wintterlin, J.; Greber, T. Comparison of electronic structure and template function of single-layer graphene and a hexagonal boron nitride nanomesh on $\mathrm{Ru}(0001)$. Phys. Rev. B 2009, $79,045407$.

(41) Goriachko, A.; Zakharov, A. A.; Over, H. Oxygen-etching of h$\mathrm{BN} / \mathrm{Ru}(0001)$ nanomesh on the nano- and mesoscopic scale. J. Phys. Chem. C 2008, 112, 10423-10427.

(42) Goriachko, A.; He, Y.; Knapp, M.; Over, H. Self-assembly of a hexagonal boron nitride nanomesh on $\mathrm{Ru}(0001)$. Langmuir 2007, 23, 2928-2931.

(43) Preobrajenski, A. B.; Vinogradov, A. S.; Ng, M. L.; Ćavar, E.; Westerström, R.; Mikkelsen, A.; Lundgren, E.; Mårtensson, N. Influence of chemical interaction at the lattice-mismatched h-BN/ $\mathrm{Rh}(111)$ and $\mathrm{h}-\mathrm{BN} / \mathrm{Pt}(111)$ interfaces on the overlayer morphology. Phys. Rev. B 2007, 75, 245412.

(44) Morscher, M.; Corso, M.; Greber, T.; Osterwalder, J. Formation of single layer h-BN on $\mathrm{Pd}(111)$. Surf. Sci. 2006, 600, 3280-3284.

(45) Müller, F.; Hüfner, S.; Sachdev, H.; Laskowski, R.; Blaha, P.; Schwarz, K. Epitaxial growth of hexagonal boron nitride on $\mathrm{Ag}(111)$. Phys. Rev. B 2010, 82, 113406.

(46) Ćavar, E.; Westerström, R.; Mikkelsen, A.; Lundgren, E.; Vinogradov, A. S.; Ng, M. L.; Preobrajenski, A. B.; Zakharov, A. A.; Mårtensson, N. A single h-BN layer on $\operatorname{Pt}(111)$. Surf. Sci. 2008, 602, $1722-1726$.

(47) Schulz, F.; Drost, R.; Hämäläinen, S. K.; Demonchaux, T.; Seitsonen, A. P.; Liljeroth, P. Epitaxial hexagonal boron nitride on Ir(111): a work function template. Phys. Rev. B 2014, 89, 235429.
(48) Orlando, F.; Larciprete, R.; Lacovig, P.; Boscarato, I.; Baraldi, A.; Lizzit, S. Epitaxial growth of hexagonal boron nitride on $\operatorname{Ir}(111)$. J. Phys. Chem. C 2012, 116, 157-164.

(49) Joshi, S.; Ecija, D.; Koitz, R.; Iannuzzi, M.; Seitsonen, A. P.; Hutter, J.; Sachdev, H.; Vijayaraghavan, S.; Bischoff, F.; Seufert, K.; et al. Boron nitride on $\mathrm{Cu}(111)$ : an electronically corrugated monolayer. Nano Lett. 2012, 12, 5821-5828.

(50) Preobrajenski, A. B.; Vinogradov, A. S.; Mårtensson, N. Monolayer of $\mathrm{h}-\mathrm{BN}$ chemisorbed on $\mathrm{Cu}(111)$ and $\mathrm{Ni}(111)$ : the role of the transition metal 3d states. Surf. Sci. 2005, 582, 21-30.

(51) Grad, G. B.; Blaha, P.; Schwarz, K.; Auwärter, W.; Greber, T. Density functional theory investigation of the geometric and spintronic structure of $\mathrm{h}-\mathrm{BN} / \mathrm{Ni}(111)$ in view of photoemission and STM experiments. Phys. Rev. B 2003, 68, 085404.

(52) Shimoyama, I.; Baba, Y.; Sekiguchi, T.; Nath, K. G. A theoretical interpretation of near edge $\mathrm{X}$-ray absorption fine structure of hexagonal boron nitride monolayer on $\mathrm{Ni}(111)$. J. Electron Spectrosc. Relat. Phenom. 2009, 175, 6-13.

(53) Koch, H. P.; Laskowski, R.; Blaha, P.; Schwarz, K. Adsorption of small gold clusters on the h-BN/Rh(111) nanomesh. Phys. Rev. B 2012, 86, 155404.

(54) Koch, H. P.; Laskowski, R.; Blaha, P.; Schwarz, K. Adsorption of gold atoms on the h-BN/Rh(111) nanomesh. Phys. Rev. B 2011, 84, 245410.

(55) Widmer, R.; Passerone, D.; Mattle, T.; Sachdev, H.; Gröning, O. Probing the selectivity of a nanostructured surface by xenon adsorption. Nanoscale 2010, 2, 502-508.

(56) Wang, B.; Bocquet, M.-L. Monolayer Graphene and h-BN on metal substrates as versatile templates for metallic nanoclusters. J. Phys. Chem. Lett. 2011, 2, 2341-2345.

(57) Natterer, F. D.; Patthey, F.; Brune, H. Ring state for single transition metal atoms on boron nitride on $\mathrm{Rh}(111)$. Phys. Rev. Lett. 2012, 109, 066101.

(58) Ma, H.; Brugger, T.; Berner, S.; Ding, Y.; Iannuzzi, M.; Hutter, J.; Osterwalder, J.; Greber, T. Nano-ice on boron nitride nanomesh: accessing proton disorder. ChemPhysChem 2010, 11, 399-403.

(59) Dong, G.; Fourré, E. B.; Tabak, F. C.; Frenken, J. W. M. How boron nitride forms a regular nanomesh on $\mathrm{Rh}(111)$. Phys. Rev. Lett. 2010, 104, 096102

(60) Tafreshi, S. S.; Roldan, A.; Dzade, N. Y.; de Leeuw, N. H. Adsorption of hydrazine on the perfect and defective copper (111) surface: a dispersion-corrected DFT study. Surf. Sci. 2014, 622, 1-8.

(61) Águila, J. E. C.; Cocoletzi, H. H.; Cocoletzi, G. H. A theoretical analysis of the role of defects in the adsorption of hydrogen sulphide on graphene. AIP Adv. 2013, 3, 032118.

(62) Kresse, G.; Joubert, D. From ultrasoft pseudopotentials to the projector augmented-wave method. Phys. Rev. B 1999, 59, 1758.

(63) Perdew, J. P.; Burke, K.; Ernzerhof, M. Generalized gradient approximation made simple. Phys. Rev. Lett. 1996, 77, 3865.

(64) Henwood, D.; Carey, J. D. Ab initio investigation of molecular hydrogen physisorption on graphene and carbon nanotubes. Phys. Rev. B 2007, 75, 245413.

(65) Monkhorst, H. J.; Pack, J. D. Special points for Brillouin-zone integrations. Phys. Rev. B 1976, 13, 5188.

(66) Hellmann, H. Einführung in die quantenchemie; Deuticke, Leipzig, Vienna, 1937.

(67) Feynman, R. P. Forces in molecules. Phys. Rev. 1939, 56, 340.

(68) Bader, R. Atoms in molecules: A quantum theory; Oxford University Press: Oxford, UK, 1990.

(69) Weast, R. C., Ed. Handbook of chemistry and physics, 65th ed; CRC Press: Boca Raton, FL, 1984.

(70) Jhi, S.-H.; Kwon, Y.-K. Hydrogen adsorption on boron nitride nanotubes: a path to room-temperature hydrogen storage. Phys. Rev. $B$ 2004, 69, 245407.

(71) Greber, T. Graphene and boron nitride single layers, arXiv:0904.1520v1; Handbook of nanophysics; CRC Press; Boca Raton, FL, 2010. 
(72) Tang, Q.; Bao, J.; Li, Y.; Zhou, Z.; Chen, Z. Tuning band gaps of $\mathrm{BN}$ nanosheets and nanoribbons via interfacial dihalogen bonding and external electric field. Nanoscale 2014, 6, 8624-8634.

(73) Tang, Q.; Zhou, Z.; Shen, P.; Chen, Z. Band gap engineering of $\mathrm{BN}$ sheets by interlayer dihydrogen bonding and electric field control. ChemPhysChem 2013, 14, 1787-1792.

(74) Csonka, Sz.; Halbritter, A.; Mihály, G.; Jurdik, E.; Shklyarevskii, O. I.; Speller, S.; van Kempen, H. Field and temperature induced effects in the surface modification process. J. Appl. Phys. 2004, 96, 6169. 